\title{
An unusual course of progressive multifocal leukoencephalopathy in a patient with idiopathic CD4+ T lymphocytopenia
}

\author{
Tamaki Iwase, Kosei Ojika, Eiichi Katada, Shigehisa Mitake, Hideka Nakazawa, \\ Noriyuki Matsukawa, Yasushi Otsuka, Yasukuni Tsugu, Hideki Kanai, Katsuhisa Nakajima
}

Nagoya City University Medical School, Aichi, Japan

T Iwase

K Ojika

E Katada

S Mitake

H Nakazawa

N Matsukawa

Y Otsuka

Y Tsugu

H Kanai

K Nakajima

Correspondence to: Dr Tamaki Iwase, Second Department of Internal Medicine, Nagoya City University Medical School,

1 Kawasumi, Mizuho-cho,

Mizuho-ku, Nagoya, Aichi 467-8601, Japan.

Telephone 008152853

8216; fax 0081528520849 .

Received 22 April 1997 and in revised form 19 August 1997

Accepted 29 October 1997

\section{Abstract}

A case is reported of idiopathic CD4+T lymphocytopenia with progressive multifocal leukoencephalopathy and cervical lymph node tuberculosis. A 57 year old Japanese man presented with cervical lymphadenopathy and progressive neurological deficits, and six months later he developed akinetic mutism. He had a persistent severely depressed number of circulating CD4+T lymphocytes in the absence of human immunodeficiency virus infection. T1 weighted MRI showed a diffuse decreased signal intensity limited to the white matter without mass effect. A brain biopsy specimen had a morphology similar to that of progressive multifocal leukoencephalopathy. Polyomavirus antigen was detected in the brain lesion, and viral DNA was identified in nucleated blood cells and urine. Unusually this serious medical condition has lasted for more than three years without remission. To our knowledge this is the first patient with CD4+T lymphocytopenia with progressive multifocal leukoencephalopathy, suggesting that similar opportunistic infections should be considered even in previously normal people.

(F Neurol Neurosurg Psychiatry 1998;64:788-791)

Keywords: idiopathic CD4+ T lymphocytopenia; progressive multifocal leukoencephalopathy; polyomavirus

Patients with CD4+ $\mathrm{T}$ lymphocytopenia and opportunistic infections, but without evidence of HIV infection or any other condition known to depress CD4+ T cells, have been described previously. ${ }^{1-8}$ Such cases were collected, analysed, and the following definition of idiopathic

Serial T cell subsets (cells $/ \mathrm{mm}^{3}$ )

\begin{tabular}{llrrrllr}
\hline Day/Month/Year & Lymphocytes & \multirow{2}{*}{ CD2 } & CD4 & CD8 & CD4/CD8 & CD4/CD2 & CD19 \\
\hline $22 / 12 / 1993$ & 1220 & 777 & 139 & 410 & 0.34 & 0.18 & 77 \\
$31 / 5 / 1994$ & 688 & 473 & 95 & 308 & 0.31 & 0.20 & 113 \\
$26 / 12 / 1994$ & 1568 & 1185 & 267 & 666 & 0.40 & 0.23 & 96 \\
$26 / 4 / 1995$ & 1394 & 1019 & 183 & 559 & 0.33 & 0.18 & 120 \\
$10 / 11 / 1995$ & 1725 & 1193 & 298 & 604 & 0.49 & 0.25 & 202 \\
$5 / 6 / 1996$ & 1140 & 782 & 201 & 386 & 0.52 & 0.26 & 185 \\
$9 / 12 / 1996$ & 1680 & 1164 & 272 & 598 & 0.46 & 0.23 & 215 \\
\hline
\end{tabular}

CD4+ T lymphocytopenia was proposed: a documented absolute number of $\mathrm{CD} 4+\mathrm{T}$ lymphocytes $<300 / \mathrm{mm}^{3}$ or a CD4+ cell count of $<20 \%$ of total $\mathrm{T}$ cells on two occasions, no evidence of HIV infection, and absence of any defined immunodeficiency or therapy that depresses the CD4+ T cell levels. ${ }^{2}$ In addition, patients with CD4+ T lymphocytopenia who have diseases indicative of a cellular immune deficiency were classified as having "severe unexplained HIV-seronegative immune suppression". ${ }^{3}$

Progressive multifocal leukoencephalopathy is a subacute demyelinating disease of the CNS caused by an infection with the papova group of the genus polyomavirus, especially the JC virus. ${ }^{9}$ This virus infects and persists in the renal and urinary system as an archetype virus. In an immune compromised patient the virus transforms to the progressive multifocal leukoencephalopathy type ${ }^{10}$ and enters the CNS by means of infected $B$ lymphocytes in the blood stream. ${ }^{11}$ In the CNS, the JC virus infects myelin forming oligodendrocytes and astrocytes, resulting in a serious demyelination. ${ }^{10}$ As progressive multifocal leukoencephalopathy is a complication of the compromised host, it usually progresses to death within six months; however, a few patients seem to have remissions and can survive for some years. ${ }^{9}$ In this report we describe what we think to be the first patient with CD4+ T lymphocytopenia who presented with progressive multifocal leukoencephalopathy which has lasted more than three years without remission.

\section{Case report}

A 57 year old Japanese man first noticed having dysarthria in August 1993, and developed gait abnormalities two months later. He was admitted to our hospital in October 1993. The patient had no known risk factors for HIV infection. He had been in good health, and had no past illness which suggested immunosuppression, or serious brain dysfunction. His wife and children had normal lymphocyte subsets. There was no family history of immunosuppression, malignancy, or serious infection.

On physical examination, there was evidence of cervical lymphadenopathy. The neurological 
A

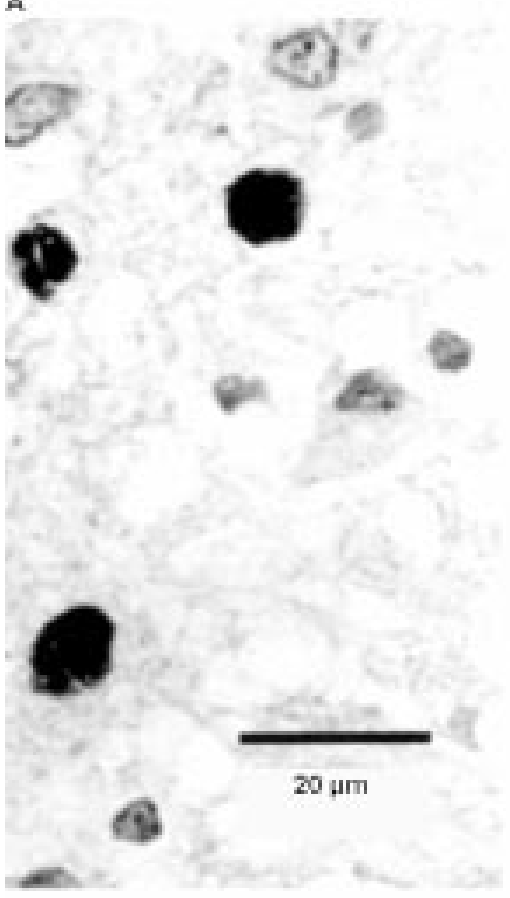

日

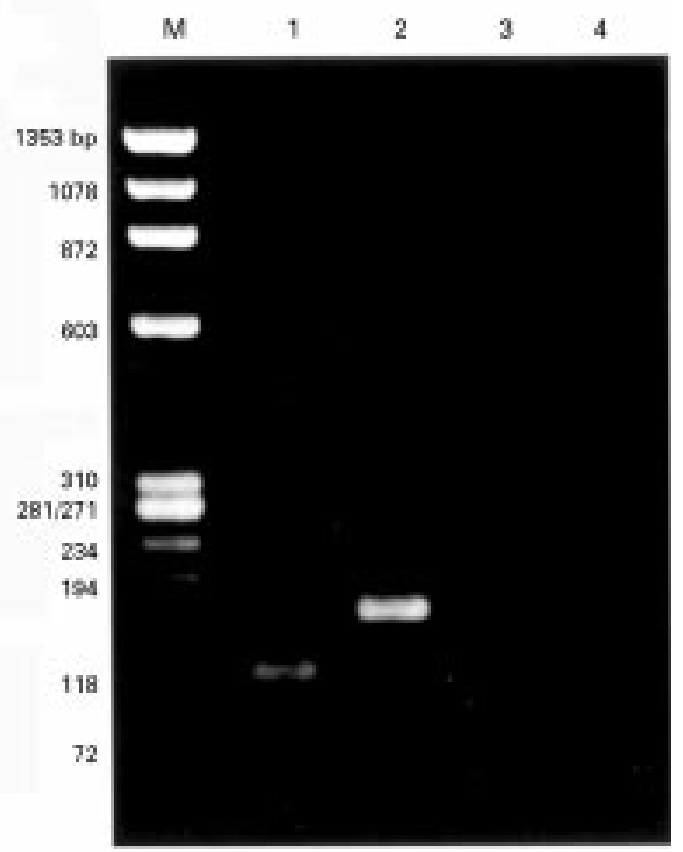

c

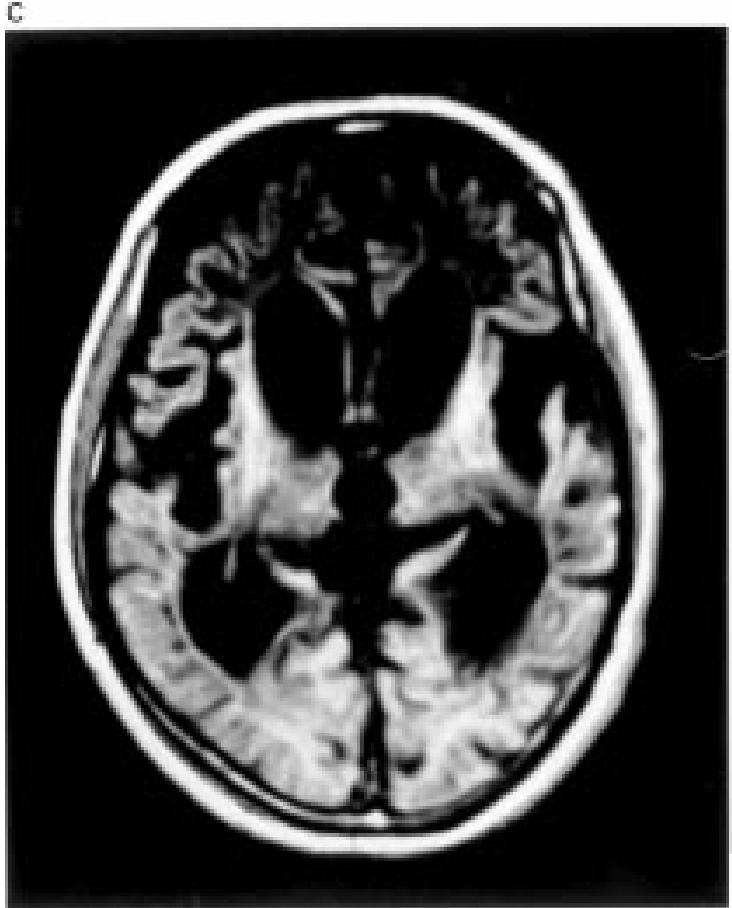

Polyomavirus infection presenting as diffuse leukoencephalopathy. (A) Histopathology of cerebellar biopsy. The immunohistochemical staining of formalin fixed, paraffin embedded 4 um thick sections for the papova virus capsid protein immunohistochemical staining of formalin fixed, paraffin embedded $4 \mu m$ thick sections for the papova virus capsid protein
$V P-1$ by using the peroxidase antiperoxidase technique showed antigen deposits in the demyelinated lesions. (B) DNA samples extracted from peripheral nucleated blood cells and urinary sediments were subjected to polymerase chain reaction using specific oligonucleotide primer pairs that amplify the early region of the polyomavirus genome that codes for large and small T antigens. A single set of 20 base oligonucleotide primers (PEP-1; 5' AGTCTTTAGGGTCTTCTACC 3', and PEP-2; 5' GGTGCCAACCTATGGAACAG 3') that are complementary to a region of DNA where $7 C$ virus and BK virus have an identical nucleotide sequence and therefore are capable of amplifying both $\mathcal{F} C$ virus and $B K$ virus DNAs, and a 40 nucleotide sequence for each virus ( $\mathcal{F} E P-1$ for $\mathcal{F C}$ virus; 5 'CTTTTTAGGTGGGGTAGAGTGTTGGGATCCT GTGTTTTCA3' and BEP-1 for BK virus; 5'TTTTTTGGGTGGTGTTGAGTGTTGAGAATCTGCTGTTGCT3') as a virus specific primer were used. $\mathcal{F E P - 1}$ or BEP-1 was used in the seminested polymerase chain reaction. The $\mathcal{F} C$ virus sequence targeted for amplification contained a BamHI site, whereas the BK virus did not. Analysis of the polymerase chain reaction products by agarose gel electrophoresis and their sensitivity to the BamHI disclosed the presence of $\mathcal{F C}$ virus DNA. Lane 1: $\mathcal{F E P - 1}$ and BamHI, lane 2: $\mathscr{F E P - 1}$ only, lane 3: BEP-1 and BamHI, lane 4: BEP-1 only, lane M: X174/HaeIII DNA digest (size marker). (C) T1 weighted (TE 15, TR 578) MRI obtained on a 1.5-tesla superconducting magnet shows the lesion characterised by a large area of decreased signal intensity limited to the white matter, without mass effect. In Fuly 1996, the lesion with severe brain atrophy extends from the brainstem and cerebellum to the periventricular and subcortical white matter and corticosubcortical junction. 
examination disclosed that he was mildly confused, and had ataxia and mild bilateral weakness of the arms and legs. The reflexes were brisk with a positive Babinski's sign on both sides.

Chest radiography showed neither consolidation nor pleural effusions or lymphadenopathy. A cervical lymph node biopsy disclosed tuberculous granuloma with caeseous necrosis. The laboratory tests, comprising activities of adenosine deaminase, purine nucleoside phosphorylase, and angiotensin converting enzyme, $\beta 2$-microglobulin concentration, immunoglobulins in the serum, all autoantibodies tested, and other routine tests on the blood as well as serological virology investigations were all within normal levels. Total lymphocytes and their subsets were measured with monoclonal antibodies using flow cytometry. The absolute number of circulating CD4+ $\mathrm{T}$ lymphocytes remained persistently and severely depressed (table). The natural killer cell function tested by the standard ${ }^{51} \mathrm{Cr}$-release cytotoxic assay was found to be impaired. The lymphocyte transformation response to $\mathrm{T}$ cell mitogens, such as concanavalin A and phytohaemagglutinin, was depressed, and a skin test to the purified protein derivative tuberculin was negative. Blood tests for HIV type 1 (HIV-1) or 2 and human $\mathrm{T}$ cell lymphotropic virus type $\mathrm{I}$, were examined by the enzyme linked immunosorbent assay, western blot analysis, and polymerase chain reaction for HIV-1 proviral DNA. All negative, and have remained so to date.

An EEG showed diffuse slow waves, and no periodic synchronised discharges were repeatedly recorded throughout his clinical course. CT obtained in December 1993 disclosed a unifocal hypodensity of the brainstem and cerebellar white matter, but no mass effect or contrast enhancing effect were found. Brain MRI showed the lesion limited to the white matter, as characterised by the area of increased signal intensity on T2 weighted MRI and a decreased signal intensity on the T1 weighted MRI. Analysis of CSF showed normal protein concentrations, no pleocytosis, and an increased myelin basic protein concentration (144 ng/ml, normal range: $<4 \mathrm{ng} / \mathrm{ml}$ ), whereas oligoclonal bands were not detected. The laboratory tests on serum, blood cells, urine, and $\mathrm{CSF}$ as well as further clinical examinations to establish the origin of the lesion failed to show metabolic diseases, common CNS infections, or cerebrovascular diseases. To verify the nature of the brain lesion, a biopsy of the left cerebellum tissue was obtained in January 1994. The light microscopical examination disclosed that the area of extensive demyelination had a mass infiltration of numerous fat laden macrophages. The increased numbers of reactive and bizarre hypertrophic astrocytes were similar to the characteristic giant astrocytes of progressive multifocal leukoencephalopathy. Although no swollen oligodendrocytes with intranuclear inclusions or infiltration of mononuclear cells were found, papova virus capsid protein VP-1 immunodeposits were detected in the demyelinated lesions (figure A). ${ }^{12}$ JC virus DNA was identified in
DNA samples extracted from peripheral nucleated blood cells and urinary sediments by a combination method of polymerase chain reaction amplification and restriction endonuclease analysis (figure B). ${ }^{13}{ }^{14}$

Despite treatment for tuberculosis with isonicotinic acid hydrazide, rifampicin, and streptomycin, and high dose intravenous steroid therapy (two three day courses of 500 $\mathrm{mg}$ /day methylprednisolone) with a one month interval between courses, the patient continued to deteriorate, showing severe dementia, bulbar palsy, tetraparesis, and myoclonus, and falling into a state of akinetic mutism by April 1994 . Nasogastric feeding and a tracheostomy were necessary to keep him alive. No positive $M$ tuberculosis cultures were obtained from sputum, blood, urine, or CSF, and no new lymphadenopathy had become evident over this period. Corresponding to the steady deterioration of neurological symptoms, there was a progressive enlargement of the brain lesion as found by CT and in MRI, together with severe brain atrophy, extending from the brainstem and cerebellum to the periventricular and subcortical white matter of the cerebrum, and lining the corticosubcortical junction (figure $\mathrm{C}$ ). The patient has been alive for more than three years without any improvement in his clinical state.

\section{Discussion}

Our patient manifested progressive neurological complications and subcortical white matter lesions as shown by CT and MRI. Similar diseases to progressive multifocal leukoencephalopathy affecting adulthood include Creutzfeldt-Jacob disease and related encephalopathy, leukodystrophy, cerebral malignant lymphoma, and primary brain vasculitis. Differential diagnosis of these disorders often requires a brain biopsy. At onset our patient displayed the clinicopathological features of cerebello-brain stem progressive multifocal leukoencephalopathy, ${ }^{15}$ with the detection of polyomavirus infection in his nucleated blood cells and demyelinated cerebellar tissue. Although progressive multifocal leukoencephalopathy is a fatal complication of an immune compromised host, ${ }^{9}$ a few cases have remission of the illness and survive for some years depending on the amelioration of primary diseases that perturbed the host immunity. ${ }^{16}$ In this study the patient with progressive multifocal leukoencephalopathy showed a continuous extension of the lesion as detected by imaging analysis which was coincident with clinical symptoms from the cerebello-brainstem to the entire subcortex of the cerebrum, manifesting in akinetic mutism and that has lasted more than three years. This unusual course of progressive multifocal leukoencephalopathy is ascribed to the CD4+ T lymphocytopenia as the primary immune disorder.

The aetiology of ICL remains unclear. However, two familial cases have been reported. ${ }^{4}{ }^{17}$ A recent study of "normal" persons reported a substantial proportion of subjects with CD4+ $\mathrm{T}$ lymphocytopenia, suggesting that this condition may occur as a result of the inherent 
variability in the measurement of $\mathrm{T}$ lymphocyte subsets. ${ }^{18}$ Further, it is hypothesised that CD4+ T lymphocytopenia represents the tail end of natural statistical variation in CD4+ cell counts. ${ }^{19}$ In addition, an important feature of ICL is that the number of CD4+ $\mathrm{T}$ cells remains unchanged throughout the accompanying lowering of cellular immunity as shown in this case. Thus it is conceivable that CD4+ $\mathrm{T}$ lymphocytopenia and progressive multifocal leukoencephalopathy themselves are not fatal, but the combination of both medical conditions is devastating and detrimental to the quality of life.

Several associated complications of the CNS have been reported in patients with CD4+ T lymphocytopenia or severe unexplained HIV seronegative immune suppression. They include undefined dementia and encephalopathy, ${ }^{7}$ opportunistic cryptococcal or Toxoplasma infection, ${ }^{247}$ histoplasmosis, ${ }^{2}$ epilepsy, ${ }^{2}$ and intracranial haemorrhage. ${ }^{3}$ However, to our knowledge, no patient similar to ours has been described previously. As it has been suggested that progressive multifocal leukoencephalopathy occurs in association with $4 \%$ of patients with AIDS $^{20}$ we should consider progressive multifocal leukoencephalopathy as the cause of the undefined dementia and encephalopathy in the patient with CD4+ $\mathrm{T}$ lymphocytopenia or severe unexplained HIV seronegative immune suppression.

We are indebted to Dr Yoshio Hashizume, Institute for Medical Science of Aging, Aichi Medical University, Aichi, Japan, for hi helpful comments on the histopathological findings, and to D Kenzo Kato, National Institute of Health, Tokyo, Japan, for kindly providing the anti-JCV antibody and the polymerase chain reaction primers, and to Drs Yuji Moriwaki and Tetsuya Yamamoto, third internal medicine, Hyogo College of Medicine, for kindly measuring activity of purine nucleoside phosphorylase.

1 Pankhurst C, Peakman M. Reduced CD4+ T cells and severe oral candidiasis in absence of HIV infection [letter] Lancet $1989 \cdot \mathrm{i}: 672$

2 Smith DK, Neal JJ, Holmberg SD, the Centers for Disease Control Idiopathic CD4+ T-lymphocytopenia Task Force. Unexplained opportunistic infections and CD4+ T-lymphocytopenia without HIV infection. An investigation of cases in the United States. N Engl f Med 1993;328: tion of
3 Heymann DL, Belsey E, Esparsa JG. CD4+ T-lymphocytopenia without HIV infection [letter]. N Engl f Med 1993;328:1848.

4 Seligmann M, Autran B, Rabian C, et al. Profound and possibly primary "idiopathic CD4+ T lymphocytopenia" in a patient with fungal infections. Clin Immunol Immunopathol 1994;71:203-7.

5 Laurence J, Siegal FP, Schattner E, et al. Acquired immunodeficiency without evidence of infection with human immunodeficiency virus types 1 and 2. Lancet 1992;340: 273-4.

6 Saiki O, Ogawa H, Ikeda T, et al. Acquired T cell specific deficiency other than acquired immunodeficiency syndrome (AIDS). Intern Med 1992;31:11-6.

7 Ho DD, Cao Y, Zhu T, et al. Idiopathic CD4+ T-lymphocytopenia- immunodeficiency without evidence of HIV infection. N Engl f Med 1993;328:380-5.

8 Ferrer X, Vital C, Larriviere M, et al. Idiopathic CD4+ T-cell lymphocytopenia and subacute inflammatory demyelinating polyradiculoneuropathy. Neurology 1995;45:1967.

9 Walker DL. Progressive multifocal leukoencephalopathy: an opportunistic viral infection of the nervous system. In: Vinken PJ, Bruyn GW, Klawans HL, eds. Handbook of clinical neurology. Vol 34. Infections of the nervous system, part II. Amsterdam: North-Holland, 1978:307-29.

10 Yogo Y, Kitamura T, Sugimoto C, et al. Isolation of a possible archetypal JC virus DNA sequence from nonimmunocompromized individuals. F Virol 1990;64:3139-43.

11 Houff SA, Major EO, Katz DA, et al. Involvement of JC virus-infected mononuclear cells from the bone marrow and spleen in the pathogenesis of progressive multifocal leukoencephalopathy. $N$ Engl f Med 1988;318:301-5.

12 Shimada H, Noda K, Mori M, et al. Papovavirus detection by electron microscopy in the brain of an elderly patient without overt progressive multifocal leukoencephalopathy. Virchows Arch 1994;424:569-72.

13 Mori M, Aoki N, Shimada H, et al. Detection of JC virus in the brains of aged patients without progressive multifocal leukoencephalopathy by the polymerase chain reaction and Southern hybridization analysis. Neurosci Lett 1992;141: 151-5.

14 Arthur RR, Dagostin S, Shah KV. Detection of BK virus and JC virus in urine and brain tissue by the polymerase chain reaction. f Clin Microbiol 1989;27:1174-9.

15 Takahashi H, Yogo Y, Furuta Y, et al. Molecular characterization of a JC virus (Sap-1) clone derived from a cerebellar form of progressive multifocal leukoencephalopathy. Acta Neuropathol 1992;83:105-12.

16 Price RW, Nielsen S, Horten B, et al. Progressive multifocal leukoencephalopathy: a burn-out case. Ann Neurol 1983; 13:485-90.

17 Wakeel RAP, Urbaniak SJ, Armstrong SS, et al. Idiopathic CD4+ lymphocytopenia associated with chronic pruritic papules. Br f Dermatol 1994;131:371-5.

18 Sheppard $\mathrm{H}$, Winkelstein W, Lang W, et al. CD4+ T-lymphocytopenia without HIV infection [letter]. $N$ Engl f Med 1993;328:1847-8.

19 Reichert T. Idiopathic CD4+ T-lymphocytopenia: a quantiative view. Lancet 1993;341:636

20 Berger JR, Kaszovitz B, Post MJD, et al. Progressive multifocal leukoencephalopathy associated with human immunodeficiency virus infection. Ann Int Med 1987;107: $78-87$. 